Proceedings of the 2006 Winter Simulation Conference

L. F. Perrone, F. P. Wieland, J. Liu, B. G. Lawson, D. M. Nicol, and R. M. Fujimoto, eds.

\title{
VISSIM: A MULTI-PARAMETER SENSITIVITY ANALYSIS
}

\author{
Nicholas E. Lownes \\ Dept. of Civil, Architectural and Environmental \\ Engineering \\ 1 University Station, C1761 ECJ 6.900 \\ The University of Texas at Austin \\ Austin, TX 78712
}

\author{
Randy B. Machemehl, P.E. \\ Dept. of Civil, Architectural and Environmental \\ Engineering \\ 1 University Station, C1761 ECJ 6.908 \\ The University of Texas at Austin \\ Austin, TX 78712
}

\begin{abstract}
Traffic microsimulation is increasingly a preferred method of traffic analysis for today's transportation professionals. The importance of properly calibrating these traffic simulations is evidenced by the adoption of microsimulation calibration standards by several state and federal transportation authorities. A component of the calibration process is the calibration of the simulation for capacity. Capacity is a high-level measurement that is a function of many lowerlevel user-defined input parameters. VISSIM utilizes psychophysical car-following models that rely on ten userdefined parameters to represent freeway driving behavior. Several VISSIM driver behavior parameters have been shown to have a significant impact on roadway capacity. This paper seeks further understanding of the performance of the VISSIM traffic microsimulator by investigating the impact of driver behavior parameter combinations on a measure of freeway capacity. This paper is intended to provide insight useful for manual calibration of VISSIM microsimulation or the development of calibration algorithms.
\end{abstract}

\section{INTRODUCTION}

Traffic microsimulation is increasingly a preferred method of traffic analysis for today's transportation professionals. The importance of properly calibrating these traffic simulations is evidenced by the adoption of microsimulation calibration standards by several state and federal transportation authorities, most of which parallel standards adopted by the Federal Highway Administration (FHWA) in 2004 (Dowling, Skabardonis and Alexiadis 2004). Calibration for capacity is considered the first of three steps in a recommended calibration strategy by FHWA. The recommended calibration process not described in detail in this paper, is impacted by the information presented here. The FHWA guidelines acknowledge that the calibration of a high-level measurement, such as capacity, using lower- level parameters is a process that may not provide a global optimal solution. Often there are multiple parameter configurations that can provide optimal solutions or a particular set of parameter values are optimal only for one location within the simulation and produce unrealistic results at other locations. In these situations the simulation developer must rely on personal knowledge of the simulation and simulated environment, calibration parameter optimization software, or both.

When calibrating for capacity, greater understanding of the parameters that impact simulated capacity will help to address situations in which multiple parameter combinations produce optimal solutions and in which optimal solutions are location-specific. This greater depth of understanding will aid the simulation developer in directly addressing calibration issues or properly selecting optimization tools that match and exploit the properties of the calibration problem and the attributes of the selected optimization methodology.

VISSIM, a discrete, stochastic, time step based microscopic traffic flow simulation model has become increasingly popular throughout the world. VISSIM utilizes the car-following model for freeway travel based on the work of Wiedemann $(1974,1991)$ as described by Panwai and Dia (2005). The car-following behavior of vehicles in a VISSIM freeway simulation is modified through ten driver behavior parameters (labeled CC0 - CC9) that represent different aspects of four assumed driving modes: freedriving, approaching, following, and braking.

Lownes and Machemehl (2006) investigated the impact of individual components on simulation capacity for a stretch of freeway in the Dallas, Texas metropolitan area. Several of the driver behavior parameters were found to exert significant influence on the capacity of the roadway individually. Details of these parameters will be discussed in a later section. Fellendorf and Vortisch (2001) analyzed the car-following model empirically in American and German contexts in validating the car-following model theory for use in Germany and abroad. However, their re- 


\section{Lownes and Machemehl}

search was not intended to look at the impact of the carfollowing model on simulated capacity. Its intent was the validation of the car-following model itself.

Gomes, May, and Horowitz (2004) developed and calibrated a VISSIM model for a congested freeway and investigate the relative impacts of several driver behavior parameters. The authors describe the influence of chosen tuning parameters as they relate to the calibration of the simulation model. These tuning parameters include several of the driver behavior parameters investigated in this study. Several other studies have investigated the performance of VISSIM compared to other popular traffic microsimulation packages. Moen et al. (2000), Bloomberg and Dale (2000), and Tian et al. (2002) all investigated the performance of VISSIM by comparing it to CORSIM, a popular traffic microsimulator developed by FHWA that has been studied extensively over the past 30 years, and to which VISSIM compared favorably.

From these previous studies a better understanding of the car-following model itself, the performance of VISSIM compared to established microsimulators, and of individual driver behavior parameter impact on simulation performance is gained. The next step in understanding how VISSIM works is the investigation of how these driver behavior parameters interrelate and how their relationships affect the simulated capacity.

\section{BACKGROUND}

As with nearly any investigation into high-level characteristics of a simulation the results are limited in interpretation by the simulation used in the evaluation, the means of comparison and evaluation, and the definition of key concepts used in the study. This section seeks to briefly describe and clarify these aspects of the study to aid in the interpretation of the results and help in identifying any areas for improvement in future work.

\subsection{Definition of Capacity}

May (1990) describes bottleneck formation as the point at which flow is no longer equal to demand, but is limited by the roadway capacity. Therefore, the flow immediately downstream of the bottleneck formation point can be considered a measure of the capacity of that particular section of roadway. The roadway capacity in this study is considered the observed queue discharged in one hour immediately downstream of the bottleneck formation point on a single date during the evening peak period. The simulation attempts to replicate a single occurrence of congestion in an attempt to later isolate the impacts of the simulation parameters from the variation of the volume data; therefore, only one day of volume data is used in the construction of the simulation. Observed capacity values are obtained using manual counts of video tapes of the bottleneck forma- tion point, while simulation capacity is estimated using the VISSIM traffic counting tool immediately downstream of the same (simulated) bottleneck formation point. This definition of capacity is supported by the guidelines adopted by FHWA (Dowling, Skabardonis and Alexiadis 2004).

The simulation, data, and definition of capacity are the same as those used in the earlier study by Lownes and Machemehl (2006) when impacts of driver behavior parameters were investigated individually.

\subsection{Simulation Description}

As noted previously, the simulation used in this study is identical to that which was used in a previous study. The selected bottleneck location for simulation is the interchange of US $75 \mathrm{NB}$ and SH190 near the cities of Plano and Richardson, Texas. The US 75 - SH190 interchange is a directional interchange that was constructed in the mid 1990's. SH190 has three full lanes in each (E-W) direction while US 75 has four full lanes in each (N-S) direction. The bottleneck to be simulated forms at the weaving section on US 75 northbound created by the SH190 and the 15th Street exit.

The simulation created for this interchange includes only freeway links and does not consider the routing impacts of congestion on the surrounding network. It is intended that by maintaining a simpler representation of the US 75 corridor the impacts of driver behavior parameters can be better isolated and will not be influenced, enhanced, or diluted by other network complexities. The simplicity of the simulation developed for the US 75 corridor at the SH 190 interchange also allowed for the calibration of the model with only very minor modifications to the driver behavior parameters prior to the sensitivity analysis, and these modifications were in line with the previous efforts of Gomes, May, and Horowitz (2004).

\subsection{Significant Driver Behavior Parameters}

Following are brief descriptions of those parameters that were found to have a significant impact on roadway capacity by Lownes and Machemehl (2006)

\subsubsection{CC0 - Stopped Condition Distance}

$\mathrm{CC} 0$ is the distance (or clear space) that a driver wishes to maintain behind a stopped vehicle on a freeway. CCO is influential in capacity calculation as it is used to calculate the safety distance maintained by drivers in conjunction with CC1 using the following equation from the VISSIM User's Manual (PTV 2004), where $d x \_s a f e=C C 0+C C 1$ * speed $(\mathrm{ft} / \mathrm{s})$ is defined as the clear space desired by the driver. The default value of CCO is 4.92 feet. 


\section{Lownes and Machemehl}

\subsubsection{CC1 - Headway Time}

$\mathrm{CC} 1$ is the factor shown in the equation above that controls the speed dependent portion of the safety distance desired by drivers. $\mathrm{CC} 1$ can certainly be expected to impact roadway capacity as it directly and significantly alters the headways (or at least the mean of the headways) that are maintained by drivers. The default value of $\mathrm{CC} 1$ is 0.90 seconds.

\subsubsection{CC2 - 'Following' Variation}

$\mathrm{CC} 2$ is a factor which restricts the longitudinal oscillation of vehicles in the simulation. The longitudinal oscillation refers to the distance increment beyond the safety distance (dx_safe) that a driver will allow between vehicles before the driver intentionally moves closer to the followed vehicle. The default value is $13.12 \mathrm{ft}$.

\subsubsection{CC4 \& CC5 - 'Following' Thresholds}

CC4 and CC5 are parameters that control the upper and lower following thresholds (the sensitivity to deceleration and acceleration of preceding car) in the driver behavior model, respectively. Smaller absolute values of CC4 and $\mathrm{CC} 5$ result in a more sensitive reaction of drivers to the accelerations and decelerations of the preceding car (PTV 2004). Therefore, for smaller absolute values of CC4 (the negative value) and CC5 (the positive value), the vehicles are more tightly coupled as they move throughout the simulation. The default values of $\mathrm{CC} 4 / \mathrm{CC} 5$ are $+/-0.35$.

\subsubsection{CC8 - Stopped Condition Acceleration}

CC8 is a parameter affecting acceleration from a stopped condition. $\mathrm{CC} 8$ is necessarily bounded by the maximum and minimum acceleration values defined as base data functions in the VISSIM program. Therefore, any values of acceleration assigned by CC 8 above those defined as maximums for a vehicle will be ignored and the simulation is run with the maximum theoretical acceleration. The default value is $11.48 \mathrm{ft} / \mathrm{s}^{2}$ which coincides with the maximum allowable acceleration for passenger cars.

\section{PARAMETER COMBINATIONS}

Six parameter combinations are considered in this analysis and one parameter is added to those that were found to provide significant effects in the previous study. The additional parameter, $\mathrm{CC} 7$, is also considered in three of the combinations. CC7 is defined as the actual acceleration rate during the oscillation process, that is, this parameter represents what acceleration the driver desires during oscillation separate from the vehicle capabilities. While this parameter did not significantly influence the capacity of the simulation in the previous study, it is considered plausible that $\mathrm{CC} 7$ represents a measure of driver aggressiveness that may not be captured by other parameters and while having little impact individually, may influence the impacts of other parameters. It is for this interaction that $\mathrm{CC} 7$ is included in this analysis.

The parameter combinations are evaluated at five levels of each parameter, resulting in 25 parameter pairs evaluated for each combination. The five levels of each parameter are determined by establishing a range bounded by one-third the calibrated and three times the calibrated value, these endpoints serving as two of the five values. The calibrated value serves as one value and the remaining two values are approximate midpoints between the calibrated value and the bounds. Each pair is observed in six replicate runs, resulting in 150 total observations for each combination.

\section{1 $\mathrm{CCO}$ and $\mathrm{CC} 8$}

This combination is analyzed because a relationship between stopped condition acceleration and stopped condition distance is logically plausible. For example, setting the stopped condition distance at a very small value may result in a much different change in capacity with changes in stopped condition acceleration than a large stopped condition distance. This difference could be due to the particular way in which the driver behavior parameters interact or other safety thresholds being approached by particular parameter combinations. The values of $\mathrm{CC} 0$ evaluated are: $2,5,8,12$, and 15 . The values of CC8 evaluated are: $4,12,20,28$, and 35 .

\section{$3.2 \mathrm{CC} 1$ and $\mathrm{CC} 4 / \mathrm{CC} 5$}

The desired headway time and the sensitivity of drivers to the preceding vehicle could potentially have an interaction effect that needs to be accounted for in the calibration process. Logically, different impacts on capacity among values of sensitivity could be expected for different values of desired headway. A lower headway time value may produce a different impact on capacity for a given sensitivity than a higher headway time and perhaps in the real world driver behavioral safety thresholds or limits on maneuverability may limit the impact that can be observed in the roadway capacity. The values of $\mathrm{CC} 1$ investigated are: $0.30,0.90,1.65,2.40$, and 3.0. The values of CC4/CC5 that are investigated are: $0.1,0.5,1.0,1.5$, and 2.0.

\subsection{CC2 and $\mathrm{CC} 4 / \mathrm{CC} 5$}

It is considered reasonable that different impacts on capacity may be experienced among values of sensitivity (CC4/CC5) for varying levels of longitudinal oscillation. $\mathrm{CC} 2$ can be considered a measure of driver aggressiveness 


\section{Lownes and Machemehl}

and it has been shown to have a significant impact on capacity. Therefore this investigation examines whether the impact from CC2 is the same across all levels of sensitivity. The values of CC2 considered are: 4, 13, 21, 30, and 39. The values of $\mathrm{CC} 4 / \mathrm{CC} 5$ are the same as previously investigated.

\section{$3.4 \mathrm{CC} 7$ and $\mathrm{CC} 2$}

CC7 and CC2 both appear to represent measures of driver aggressiveness and an investigation into the interaction (if any) between these two parameters and their impact on capacity may provide insight into how to best represent driver aggressiveness in calibrated VISSIM simulations. The values evaluated for both of these parameters are the same as those values in previous combinations in which they are involved.

\subsection{CC7 and CC4/CC5}

As CC7 is also a measure of driver aggressiveness, an investigation similar to that for $\mathrm{CC} 2$ and $\mathrm{CC} 4 / \mathrm{CC} 5$ is undertaken in a continuing effort to understand the impact and interaction of driver aggressiveness parameters on simulated capacity. The values evaluated for both of these parameters are the same as those values in previous combinations.

\section{6 $\mathrm{CC} 7$ and $\mathrm{CC} 8$}

Stopped condition acceleration, CC8, logically has a significant impact on capacity as measured in this study: the queue discharge over an hour of simulation time from the formation point of a bottleneck. It is desirable to understand whether this significant impact is the same for all levels of driver aggressiveness. The values evaluated for both of these parameters are the same as those values in previous

\subsection{Analysis Method}

A two-way complete model is used in the analysis of variance (ANOVA) for each of the parameter combinations with an $\alpha$-level of 0.05 . Since the levels of each of the parameters were pre-selected each of the parameters is considered a fixed effect. SPSS statistical software is used for the analysis. Details of the model and associated assumptions are not included in this paper to conserve space, though are available upon request.

\section{RESULTS}

Each parameter combination will have an interaction plot displaying the mean capacity for each pair of values and a discussion of the statistical results of the study along with a practical interpretation. The numerical results of the twoway ANOVA can be found in the Appendix.

\subsection{CCO and CC8}

The interaction plot in Figure 1 displays the relationship between capacity and $\mathrm{CC} 8$ for the five values of $\mathrm{CC} 0$ selected for analysis. As discussed earlier, the maximum value of $\mathrm{CC} 8$ is overridden by the maximum allowable acceleration given by separate base data in VISSIM. The relatively unchanging capacity experienced by values of CC8 greater than 12 coincides with this aspect of VISSIM.

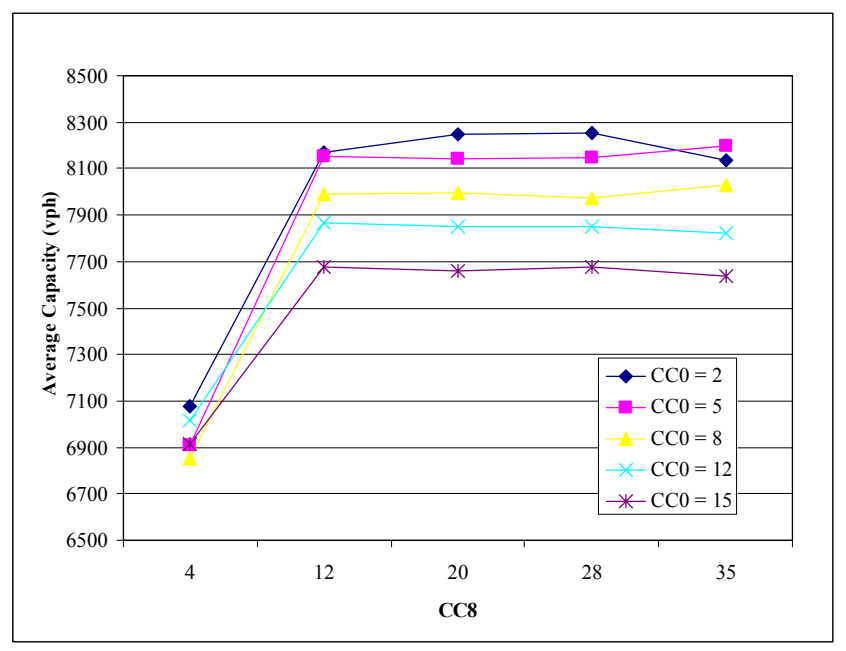

Figure 1: Stopped Condition Distance (CC0) vs. Stopped Condition Acceleration (CC8) Interaction Plot

The Two-way ANOVA results support the results of the previous study, both $\mathrm{CC} 0$ and $\mathrm{CC} 8$ significantly impact capacity. In addition to this reinforcement, the interaction term for $\mathrm{CC} 0 * \mathrm{CC} 8$ was found to be significant at the $\alpha$ level of 0.05 . Looking again at Figure 1, interaction is apparent in both the $4-12$ and $28-35$ ranges of CC8. Since the $28-35$ range is curtailed by the maximum value of allowable acceleration $\left(11.5 \mathrm{ft} / \mathrm{s}^{2}\right)$ the $4-12$ range will be concentrated upon in practical interpretation, while the statistical significance of the interaction term applies to the entire range of $\mathrm{CC} 8$. A potential explanation of the interaction at values of $\mathrm{CC} 8$ greater than the allowable acceleration could be evidence of the instability of the simulation when extreme values of a behavior parameter are used in the simulation. In future study, similar analysis should be undertaken investigating the relationship of CC8 only for values of CC8 under the maximum allowable acceleration value to better understand the relationship and the source of the interaction component variation.

The most drastic difference in capacity impact among the values of $\mathrm{CC} 0$ is observed between values 5 and 15 of $\mathrm{CC} 0$. The capacity of the roadway is 6909 and $6917 \mathrm{vph}$ for $\mathrm{CC} 0=5 \mathrm{ft}$ and $\mathrm{CC} 0=15 \mathrm{ft}$, respectively at $\mathrm{CC} 8=4$ 


\section{Lownes and Machemehl}

$\mathrm{ft} / \mathrm{s}^{2}$. When CC8 increases to $12 \mathrm{ft} / \mathrm{s}^{2}$ the simulations with $\mathrm{CC} 0=5 \mathrm{ft}$ experience an increase in average capacity to 8155 vph while the simulations with $\mathrm{CC} 0=15 \mathrm{ft}$ only see an increase of average capacity to $7676 \mathrm{vph}$, an impact 500 vph lower than the simulations with $\mathrm{CC} 0=5$.

The interaction plot suggests that in general, as the value of $\mathrm{CCO}$ increases, the impact on capacity due to an increase in $\mathrm{CC} 8$ from $4-12 \mathrm{ft} / \mathrm{s}^{2}$ decreases. As the stopped condition distance increases, the impact on capacity from increasing stopped condition acceleration decreases.

\subsection{CC1 and $\mathrm{CC} 4 / \mathrm{CC5}$}

The interaction plot in Figure 2 displays the relationship between capacity and CC4/CC5 for the five values of CC1 selected for analysis.

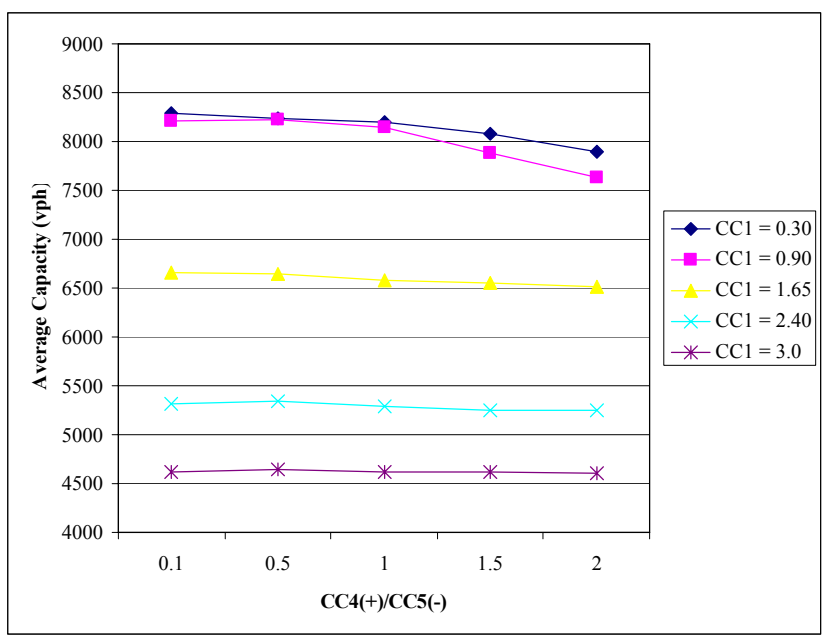

Figure 2: Headway Time (CC1) vs. Following Thresholds (CC4/CC5) Interaction Plot

The Two-way ANOVA results support the results of the previous study, both $\mathrm{CC} 1$ and $\mathrm{CC} 4 / \mathrm{CC} 5$ significantly impact capacity. In addition, the interaction term for this combination was also found to be significant at the $\alpha$-level of 0.05 . The interaction between $\mathrm{CC} 1$ and $\mathrm{CC} 4 / \mathrm{CC} 5$ is most apparent in the CC4/CC5 range of $1-2$. It is apparent in Figure 2 that as $\mathrm{CC} 1$ increases the impact of increasing $\mathrm{CC} 4 / \mathrm{CC} 5$ is lessened as the line for $\mathrm{CC} 1=3.0$ is nearly flat, whereas $\mathrm{CC} 1=0.90$ experiences a reduction in capacity of over $500 \mathrm{vph}$ in that same range..

$\mathrm{CC} 1$ is the headway time that a driver wishes to maintain, in seconds, at any speed. CC4/CC5 represents the sensitivity to the accelerations and decelerations of the preceding car, with the larger absolute values representing less sensitive behavior. As the desired headway time increases the impact of a reduction in sensitivity is decreased. This result makes logical sense. As the headway time is increased, the capacity of the roadway, inversely propor- tional to the headway, is dominated by the headway time component of the simulation. At lower levels of headway time, coupling the vehicles less tightly by decreasing sensitivity to the preceding vehicle (increasing $\mathrm{CC} 4 / \mathrm{CC} 5$ ) has a larger relative impact on the headway maintained, and therefore, the capacity of the roadway.

\subsection{CC2 and $\mathrm{CC} 4 / \mathrm{CC} 5$}

The interaction plot in Figure 3 displays the relationship between capacity and $\mathrm{CC} 4 / \mathrm{CC} 5$ for the five values of $\mathrm{CC} 2$ selected for analysis.

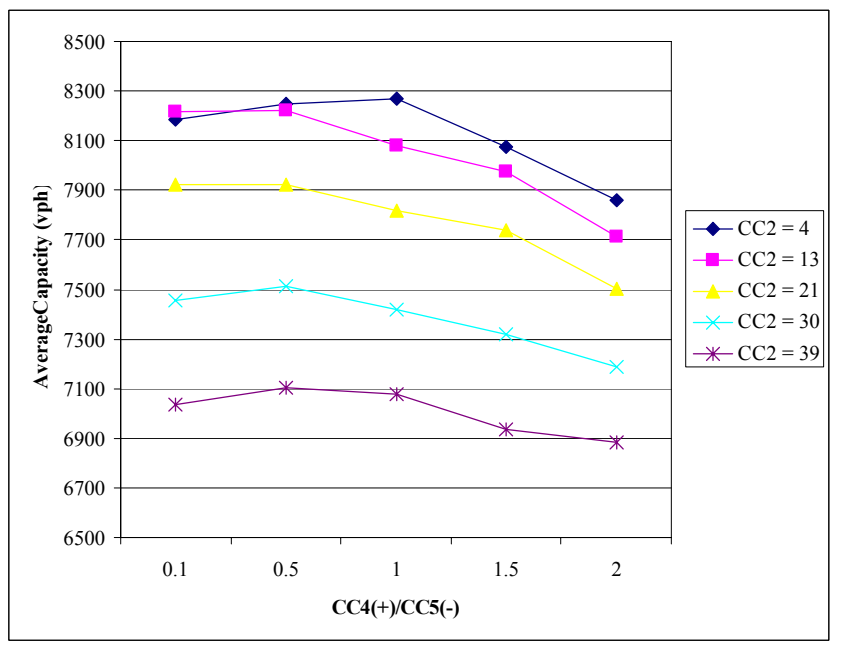

Figure 3: Following variation (CC2) vs. Following Thresholds (CC4/CC5) Interaction Plot

Consistent with the previous study, the individual impacts of CC2 and CC4/CC5 were significant at the $\alpha=0.05$ level. However, the interaction component of the analysis was found not to be statistically significant at the $\alpha=0.05$ level even though Figure 3 suggests that there may be evidence for interaction when the plots for CC2 $=4$ and $\mathrm{CC} 2$ $=13$ are scrutinized. This appearance of interaction, while statistically insignificant, may practically be an example of instability in simulation results when extreme values (here, both are at $1 / 3$ of their default value) of behavior parameters are being used. Therefore, the statistical evidence suggests that the impact on capacity of increasing or decreasing $\mathrm{CC} 4 / \mathrm{CC} 5$ (the sensitivity to the preceding driver) is the same regardless of the value of CC2 (longitudinal oscillation, the sensitivity to the desired safety distance).

The significant impacts of the two parameters individually is readily apparent in Figure 3, as is the lack of interaction of the two parameters. The slopes of the interaction plot appear approximately parallel for each value of $\mathrm{CC} 2$, suggesting that regardless which of the five values of CC2 is selected, the same capacity impact can be expected from a change to $\mathrm{CC} 4 / \mathrm{CC} 5$. 


\subsection{CC7 and CC2}

The interaction plot in Figure 4 displays the relationship between capacity and $\mathrm{CC} 2$ for the five values of $\mathrm{CC} 7 \mathrm{se}-$ lected for analysis.

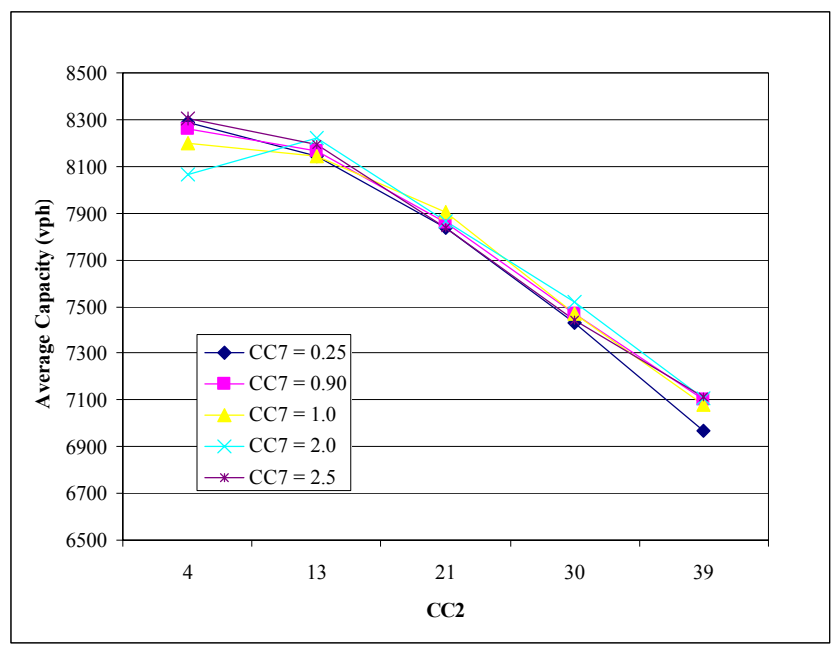

Figure 4: Oscillation Acceleration (CC7) vs. Following Variation (CC2) Interaction Plot

The results of the statistical analysis found that only the individual impact of $\mathrm{CC} 2$ was significant in its impact on simulated capacity. CC7 is one measure of driver aggressiveness in VISSIM, and it has no statistically significant influence on capacity by itself or in its interactions with $\mathrm{CC} 2$.

\subsection{CC7 and $\mathrm{CC} 4 / \mathrm{CC} 5$}

The interaction plot in Figure 5 displays the relationship between capacity and $\mathrm{CC} 4 / \mathrm{CC} 5$ for the five values of $\mathrm{CC} 7$ selected for analysis.

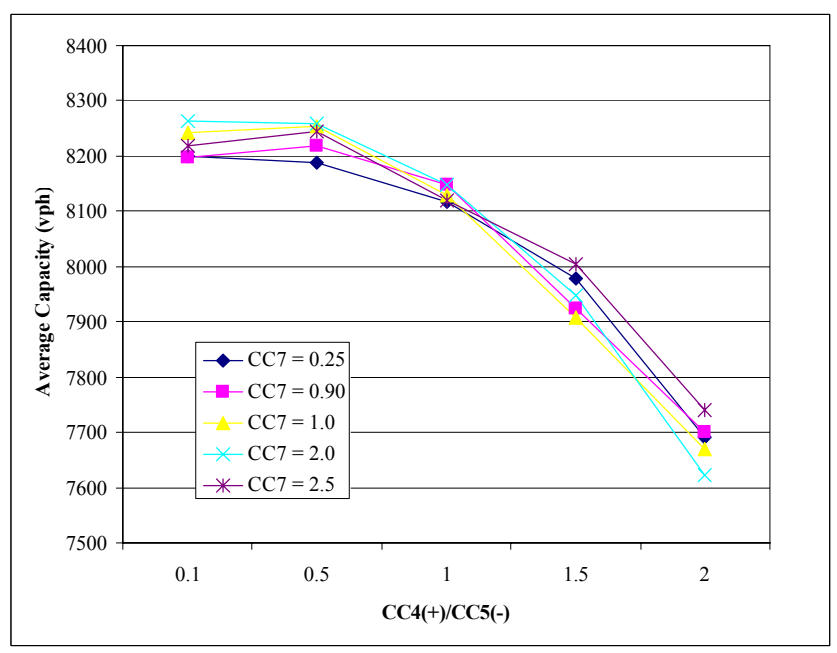

Figure 5: Oscillation Acceleration (CC7) vs. Following Thresholds (CC4/CC5) Interaction Plot
The results of the statistical analysis found that only the individual impact of CC4/CC5 was significant in its impact on simulated capacity. CC7, again, appears to have no statistically significant influence on capacity by itself or in its interactions with $\mathrm{CC} 4 / \mathrm{CC} 5$, the following thresholds which govern sensitivity to the preceding driver's acceleration and deceleration.

\subsection{CC7 and CC8}

The interaction plot in Figure 6 displays the relationship between capacity and $\mathrm{CC} 8$ for the five values of $\mathrm{CC} 7 \mathrm{se}-$ lected for analysis.

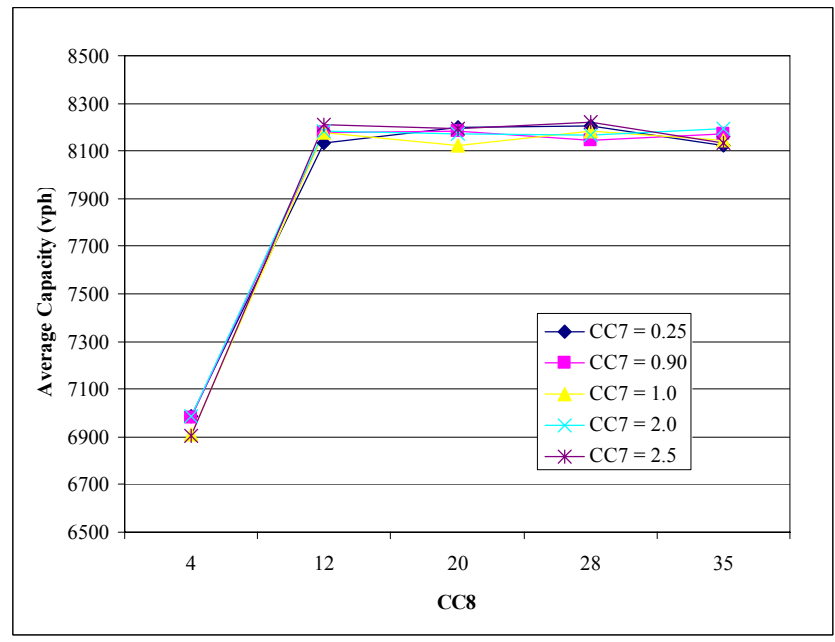

Figure 6: Oscillation Acceleration (CC7) vs. Stopped

Condition Acceleration (CC8) Interaction Plot

The results of the statistical analysis found that only the individual impact of CC8 was significant in its impact on simulated capacity. CC7 again appears to have no statistically significant influence on capacity by itself or in its interactions with $\mathrm{CC} 8$, the stopped condition acceleration.

\section{SUMMARY AND CONCLUDING REMARKS}

The primary purpose of this study was to investigate whether the impact on capacity of modifications to VISSIM driver behavior parameters is sensitive to values of other parameters. In two cases, there was interaction between driver behavior parameters: $\mathrm{CC} 0 \& \mathrm{CC} 8$, and $\mathrm{CC} 1$ \& CC4/CC5. The impact on capacity for CC8 and $\mathrm{CC} 4 / \mathrm{CC} 5$ were dependent on the values of $\mathrm{CC} 0$ and $\mathrm{CC} 1$, respectively.

This is an interesting result, as $\mathrm{CC} 0$ and $\mathrm{CC} 1$ are the two factors that are used in the calculation of $d x \_s a f e$, the VISSIM representation of minimum headway. Capacity of a roadway is so heavily dependent upon headway that when calibrating a simulation for capacity it would be simple to alter the capacity of the roadway using $\mathrm{CCO}$ and 


\section{Lownes and Machemehl}

CC1 as they have the most direct impact on headway. However, the results of this study indicate that the value of $\mathrm{CC} 0$ or $\mathrm{CC} 1$ can have a joint impact with other driver behavior parameters on capacity.

Figure 2 displays the result of interacting parameters. As an example, consider the situation in which $8000 \mathrm{vph}$ is the target capacity. In this case there are two combinations of $(\mathrm{CC} 1, \mathrm{CC} 4 / \mathrm{CC} 5)$ that would result in this capacity: $(0.90,1.25)$ and $(0.30,1.75)$. These two points may both represent optimal solutions based upon an objective function minimizing the deviation from the observed capacity. The simulation developer must select the final calibrated values based upon either knowledge of which solution best represents reality or have incorporated this knowledge into the optimization methodology.

FHWA (Dowling, Skabardonis and Alexiadis 2004) states that several optimization tools are available for use in selecting optimally calibrated parameters for microsimulation. Zhizhou, Jian, and Xiaouang (2005) applied a genetic algorithm to the calibration of VISSIM, including some driver behavior parameters. It is intended that this work will aid VISSIM users in the application of such optimization tools for calibration by helping direct heuristic methods to improve local optimal solutions or defining boundaries for specific parameters to help ensure that the final simulation best represents reality.

Future work could focus specifically on the relationships of $\mathrm{CC} 0$ and $\mathrm{CC} 1$ with the other driver behavior parameters. In addition to an analysis similar to this study, an attempt to further isolate the impacts of these two parameters on capacity and better understand their relationship quantitatively could prove useful to VISSIM users.

\section{APPENDIX: ANOVA RESULTS}

Table A-1: CC0 and CC8 ANOVA Table

\begin{tabular}{l} 
Dependent Variable: Capacity \\
\begin{tabular}{|l|c|r|r|r|r|}
\hline Source & $\begin{array}{c}\text { Type III Sum } \\
\text { of Squares }\end{array}$ & df & Mean Square & \multicolumn{1}{c|}{ F } & Sig. \\
\hline Corrected Model & $30099247.7^{\text {a }}$ & 24 & 1254135.321 & 193.729 & .000 \\
Intercept & 9054987085 & 1 & 9054987085 & 1398745 & .000 \\
CC0 & 4112584.560 & 4 & 1028146.140 & 158.820 & .000 \\
CC8 & 24976929.6 & 4 & 6244232.390 & 964.561 & .000 \\
CC0 ${ }^{*}$ CC8 & 1009733.573 & 16 & 63108.348 & 9.748 & .000 \\
Error & 809206.500 & 125 & 6473.652 & & \\
Total & 9085895539 & 150 & & & \\
Corrected Total & 30908454.2 & 149 & & & \\
\hline
\end{tabular} \\
a. R Squared $=.974$ (Adjusted R Squared $=.969)$ \\
\hline
\end{tabular}

Table A-2: CC1 and CC4/CC5 ANOVA Table

Dependent Variable: Capacity
\begin{tabular}{|l|r|r|r|r|r|}
\hline Source & $\begin{array}{c}\text { Type III Sum } \\
\text { of Squares }\end{array}$ & df & Mean Square & \multicolumn{1}{|c|}{ F } & \multicolumn{1}{c|}{ Sig. } \\
\hline Corrected Model & $302242711^{\mathrm{a}}$ & 24 & 12593446.30 & 5449.852 & .000 \\
Intercept & 6396892502 & 1 & 6396892502 & 2768275 & .000 \\
CC1 & 299989406 & 4 & 74997351.39 & 32455.333 & .000 \\
CC4CC5 & 1281562.040 & 4 & 320390.510 & 138.650 & .000 \\
CC1* CC4CC5 & 971743.627 & 16 & 60733.977 & 26.283 & .000 \\
Error & 288848.333 & 125 & 2310.787 & & \\
Total & 6699424062 & 150 & & & \\
Corrected Total & 302531560 & 149 & & & \\
\hline
\end{tabular}

a. $R$ Squared $=.999$ (Adjusted R Squared $=.999$ )

Table A-3: CC2 and CC4/CC5 ANOVA Table Dependent Variable: Capacity

\begin{tabular}{|l|r|r|r|r|r|}
\hline Source & $\begin{array}{c}\text { Type III Sum } \\
\text { of Squares }\end{array}$ & df & Mean Square & \multicolumn{1}{|c|}{ F } & Sig. \\
\hline Corrected Model & $29526185.3^{\mathrm{a}}$ & 24 & 1230257.722 & 59.040 & .000 \\
Intercept & 8817893376 & 1 & 8817893376 & 423170.2 & .000 \\
CC2 & 26456912.7 & 4 & 6614228.167 & 317.416 & .000 \\
CC4CC5 & 2751075.667 & 4 & 687768.917 & 33.006 & .000 \\
CC2 ${ }^{*}$ CC4CC5 & 318197.000 & 16 & 19887.313 & .954 & .510 \\
Error & 2604712.667 & 125 & 20837.701 & & \\
Total & 8850024274 & 150 & & & \\
Corrected Total & 32130898.0 & 149 & & & \\
\hline
\end{tabular}

Table A-4: CC7 and CC2 ANOVA Table

Dependent Variable: Capacity
\begin{tabular}{|l|r|r|r|r|r|}
\hline Source & $\begin{array}{c}\text { Type III Sum } \\
\text { of Squares }\end{array}$ & \multicolumn{1}{c|}{ df } & Mean Square & \multicolumn{1}{|c|}{ F } & \multicolumn{1}{c|}{ Sig. } \\
\hline Corrected Model & $29028208.8^{\mathrm{a}}$ & 24 & 1209508.698 & 75.854 & .000 \\
Intercept & 9031770901 & 1 & 9031770901 & 566427.3 & .000 \\
CC7 & 35745.427 & 4 & 8936.357 & .560 & .692 \\
CC2 & 28634043.1 & 4 & 7158510.773 & 448.946 & .000 \\
CC7 ${ }^{*}$ CC2 & 358420.240 & 16 & 22401.265 & 1.405 & .150 \\
Error & 1993144.333 & 125 & 15945.155 & & \\
Total & 9062792254 & 150 & & & \\
Corrected Total & 31021353.1 & 149 & & & \\
\hline
\end{tabular}

a. $\mathrm{R}$ Squared $=.936$ (Adjusted R Squared $=.923$ )

Table A-5: CC7 and CC4/CC5 ANOVA Table

\begin{tabular}{|l|c|r|r|r|r|}
\hline Dependent Variable: Capacity \\
Source & $\begin{array}{c}\text { Type III Sum } \\
\text { of Squares }\end{array}$ & \multicolumn{1}{c|}{ df } & Mean Square & \multicolumn{1}{c|}{ F } & \multicolumn{1}{c|}{ Sig. } \\
\hline Corrected Model & $6542612.333^{\mathrm{a}}$ & 24 & 272608.847 & 48.425 & .000 \\
Intercept & 9709108267 & 1 & 9709108267 & 1724670 & .000 \\
CC7 & 17922.400 & 4 & 4480.600 & .796 & .530 \\
CC4CC5 & 6415892.333 & 4 & 1603973.083 & 284.921 & .000 \\
CC7 $^{*}$ CC4CC5 & 108797.600 & 16 & 6799.850 & 1.208 & .271 \\
Error & 703693.000 & 125 & 5629.544 & & \\
Total & 9716354572 & 150 & & & \\
Corrected Total & 7246305.333 & 149 & & & \\
\hline
\end{tabular}

Table A-6: CC7 and CC8 ANOVA Table

\begin{tabular}{l} 
Dependent Variable: Capacity \\
\begin{tabular}{|l|r|r|r|r|r|}
\hline Source & $\begin{array}{c}\text { Type III Sum } \\
\text { of Squares }\end{array}$ & df & Mean Square & \multicolumn{1}{|c|}{ F } & Sig. \\
\hline Corrected Model & $35781982.2^{\mathrm{a}}$ & 24 & 1490915.924 & 148.826 & .000 \\
Intercept & 9428881414 & 1 & 9428881414 & 941208.1 & .000 \\
CC7 & 17615.773 & 4 & 4403.943 & .440 & .780 \\
CC8 & 35659817.9 & 4 & 8914954.477 & 889.907 & .000 \\
CC7 ${ }^{*}$ CC8 & 104548.493 & 16 & 6534.281 & .652 & .835 \\
Error & 1252231.167 & 125 & 10017.849 & & \\
Total & 9465915627 & 150 & & & \\
Corrected Total & 37034213.3 & 149 & & & \\
\hline
\end{tabular} \\
a. R Squared $=$ \\
\hline
\end{tabular}




\section{REFERENCES}

Bloomberg, L. and Dale, J. 2000. Comparison of VISSIM and CORSIM Traffic Simulation Models on a Congested Network. Transportation Research Record 1727, TRB, National Research Council, Washington D.C. pp. 52-60.

Dowling, R., Skabardonis, A., and Alexiadis, V. 2004. Traffic Analysis Toolbox Volume III: Guidelines for Applying Traffic Microsimulation Software. Federal Highway Administration, Report FHWA-HRT-04040 .

Fellendorf, Martin and Vortisch, Peter. 2001. Validation of the Microscopic Traffic Flow Model VISSIM in Different Real-World Situations. Available in The 80th annual meeting of the Transportation Research Board Compendium of Papers.

Gomes, Gabriel; May, Adolf and Horowitz, Roberto. 2004. A Microsimulation Model of a Congested Freeway using VISSIM. Transportation Research Record 1876, TRB, National Research Council, Washington D.C. pp. 71-81.

Lownes, N. E. and Machemehl, R. B. 2006. Sensitivity of Simulated Capacity to VISSIM Driver Behavior Parameter Modification. Forthcoming in Transportation Research Record, Transportation Research Board, National Research Council, Washington, D.C. Available in The 85th annual meeting of the Transportation Research Board Compendium of Papers.

May, Adolf D. 1990. Traffic Flow Fundamentals, Prentice Hall, Englewood Cliffs, N.J.

Moen, B., Fitts, J., Carter, D., and Ouyang, Y. 2000. A comparison of the VISSIM Model to Other Widely Used Traffic Simulation and Analysis Programs. Presented at the Institute of Transportation Engineers 2000 Annual Meeting and Exhibit, Nashville, TN.

Panwai, S. and Dia, H. 2005. Comparative Evaluation of Microscopic Car-Following Behavior. IEEE Transactions on Intelligent Transportation Systems, Vol. 6, No. 3.

PTV Planning Transport Verkehr AG. 2004. User's Manual, VISSIM 4.0, Karlsruhe, Germany.
Tian, Z. Z., Urbanik, T., Engelbrecht, R., and Balke, K. 2002. Variations in Capacity and Delay Estimates from Microscopic Traffic Simulation Models. Transportation Research Record 1802, TRB, National Research Council, Washington D.C. pp. 23-31.

Wiedemann, R. 1974. Simulation des Straßenverkehrsflusses. (In German) Schtiftenreihe des Instituts fur Verkehrswesen der Universitat Karlsruhe, Heft 8.

Wiedemann, R. 1991. Modeling of RTI-Elements on multi-lane roads. Advanced Telematics in Road Transport edited by the Commission of the European Community, vol. DG XIII.

Zhizhou, W., Jian, S., and Xiaoguang, Y. 2005. Calibration of VISSIM for Shanghai Expressway Using Genetic Algorithm. Proceedings of the 2005 Winter Simulation Conference, ed. L. F. Perrone, F. P. Wieland, J. Liu, B. G. Lawson, D. M. Nicol, and R. M. Fujimoto, $2645-2648$.

\section{AUTHOR BIOGRAPHIES}

NICHOLAS E. LOWNES is a Ph.D. candidate at The University of Texas at Austin. He holds the degrees of B.S. in Civil Engineering from Iowa State University and M.S. in Engineering from The University of Texas at Austin. His research interests include traffic engineering, traffic microsimulation, intelligent transportation systems, and transportation network analysis. His email address is <nlownes@mail.utexas.edu>.

RANDY B. MACHEMEHL is currently the Nasser I. AlRashid Centennial Professor in Transportation Engineering at The University of Texas at Austin. He earned his Ph.D. in civil engineering from The University of Texas at Austin in 1975. Dr. Machemehl also directs the Center for Transportation Research in Austin, Texas. His research interests include traffic control systems, simulation and optimization of transportation systems, psycho-physical measuring of driver behavior, and freeway bottleneck resolution. His email address is<rbm@mail.utexas.edu> 